\title{
Disease severity and renal outcomes of patients with chronic kidney disease infected with COVID-19
}

\author{
Efrat Gur ${ }^{1} \cdot$ David Levy ${ }^{1} \cdot$ Guy Topaz $^{1,2} \cdot$ Rawand Naser $^{1} \cdot$ Ori Wand $^{2,3} \cdot$ Yona Kitay-Cohen $^{1,2} \cdot$ Sydney Benchetrit ${ }^{2,4}$. \\ Erez Sarel $\left.\right|^{5} \cdot$ Keren Cohen-Hagai ${ }^{2,4}$
}

Received: 28 November 2021 / Accepted: 3 January 2022 / Published online: 1 March 2022

(c) The Author(s), under exclusive licence to The Japanese Society of Nephrology 2022

\begin{abstract}
Introduction While there is evidence of the presence of the coronavirus in the kidneys and resultant acute kidney injury (AKI), information on the effect of chronic kidney disease (CKD) on COVID-19 outcomes and its pathogenesis is currently lacking.

Methods This retrospective, observational study evaluated the outcomes of all consecutive patients hospitalized during COVID-19 outbreaks in Meir Medical Center. Serum creatinine level was assessed before hospitalization ("baseline serum creatinine") and at admission, as well as minimum and maximum serum creatinine levels during hospitalization.

Results Among 658 patients, 152 had eGFR $<60 \mathrm{ml} / \mathrm{min}$ (termed the CKD group), 506 patients served as controls. Patients in the CKD group were older, with higher prevalence of hypertension, diabetes mellitus and atherosclerosis. Disease severity and clinical presentation of CKD group were comparable to that of control group. Odds ratio for AKI was 5.8 (95\% CI 3.8-8.7; $p<0.001$ ) in CKD group vs. control group and 3.4 (95\%CI 1.1-10.8) for renal replacement therapy $(p<0.026)$. Among the CKD group, $32.2 \%$ died after COVID-19 infection versus $14.8 \%$ of the controls $(p<0.001)$. Mortality increased as CKD stage increased (14.8\% in controls, $29.6 \%$ in CKD stage 3, and 39.3\% in CKD stages 4 and 5, $p<0.001$ ).

Conclusion Despite comparable disease severity at presentation, patients with CKD had significantly more AKI events and required more renal replacement therapy during hospitalization than control patients did. Mortality increased as CKD stage increased.
\end{abstract}

Keywords Chronic kidney disease · COVID-19 outcomes · Acute kidney injury

\section{Introduction}

The coronavirus disease 2019 (COVID-19) pandemic has had an enormous impact on medical systems globally. The disease results from infection with severe acute respiratory

Keren Cohen-Hagai

keren.cohen@ clalit.org.il

1 Department of Internal Medicine C, Meir Medical Center, Kefar Sava, Israel

2 Sackler Faculty of Medicine, Tel Aviv University, Tel Aviv, Israel

3 Department of Pulmonology, Meir Medical Center, Kefar Sava, Israel

4 Department of Nephrology and Hypertension, Meir Medical Center, 59 Tchernichovsky St., 4428164 Kefar Sava, Israel

5 Department of Anesthesiology, Meir Medical Center, Kefar Sava, Israel syndrome coronavirus-2 (SARS-CoV-2) and may progress to multi-organ injury, including the kidneys. The clinical spectrum of SARS-COV-2 infection ranges from asymptomatic to critical and sometimes fatal illness. Fever, cough, myalgias, and headache are the most commonly reported symptoms. Other features, including diarrhea, sore throat, anosmia and dysgeusia, are also well-described [1-3].

Risk factors for mortality and severe COVID-19 infection include older age, male sex, hypertension, diabetes mellitus, cardiovascular disease, obesity and chronic kidney disease (CKD) [4-8]. These risk factors were also examined in a review of a cohort of over 17 million patients and found to be associated with COVID-19-related death [7]. Other factors that indicate severe infections are markers of inflammation or coagulation (fever, D-dimer level at admission, elevated fibrin degradation products, and prolonged activated partial thromboplastin and prothrombin times) [9]. 
Risk factors and risk scores to identify high-risk patients are still being developed [10-13].

While there is evidence of the presence of the coronavirus in the kidneys and resultant $\mathrm{AKI}$, the effect of CKD on COVID-19 outcomes and its pathogenesis is poorly understood $[14,15]$.

The pathogenesis of kidney damage in patients with COVID-19 involves several factors. One mechanism is the direct binding of the virus to angiotensin-converting enzyme (ACE2) on the renal parenchyma, which can explain the direct damage the virus causes to the kidney [16]. Another mechanism involves the cytokine storm after the infection, promoting tubular and endothelial dysfunction [17] and even capillary leak, which may result in disseminated intravascular coagulation [18]. Finally, the hypercoagulability state in COVID-19 infection is well known and might be complicated by tubular necrosis, inducing irreversible kidney damage [13, 19-21].

The prevalence of kidney injury in hospitalized COVID19 patients is high and is associated with higher risk of inhospital mortality [22]. A meta-analysis indicated a threefold higher likelihood of severe COVID-19 [23, 24]. CKD is associated with oxidative stress, immune dysregulation and lymphopenia $[25,26]$, which can play a role in the greater severity of COVID-19 in patients with CKD.

CKD is defined as the presence of kidney damage (usually detected as a laboratory or radiographic abnormality) or decreased kidney function (defined as estimated glomerular filtration rate [eGFR] $<60 \mathrm{~mL} / \mathrm{min} / 1.73 \mathrm{~m}^{2}$ ) for three or more months, regardless of the cause. Staging patients with CKD according to eGFR enhances risk stratification for the major complications of CKD [27, 28].

The purpose of this study was to investigate the clinical outcomes of CKD patients infected with COVID-19 infection compared to non-CKD patients (controls).

\section{Methods}

This retrospective, observational study evaluated the outcomes of patients hospitalized during COVID-19 outbreaks August 2020-December 2020, at Meir Medical Center, a 760-bed academic hospital located in Central Israel. It is the largest hospital in the district, which comprises a mostly urban population of Jews and Arabs.

Results are reported according to the STROBE statement guidelines.

\section{COVID-19 infection}

The diagnosis of SARS-CoV-2 infection was ascertained by a positive real-time reverse-transcriptase polymerase chain reaction (RT-PCR) result from nasopharyngeal swabs.

\section{Participants}

The study population included adults (age $\geq 18$ years) infected with SARS-CoV-2. All patients were hospitalized in COVID-19 wards at the medical center and were followed daily. This cohort included only non-vaccinated patients to obtain data regarding the natural history of COVID-19 and CKD

Exclusion criteria were second hospitalization, hemodialysis patients, missing medical record data and individuals after renal transplantation.

\section{Study design and kidney disease}

Serum creatinine level was assessed before admission to the hospital (baseline serum creatinine) and at admission. Minimum and maximum levels during hospitalization were also obtained and recorded for each patient.

Clinical, laboratory and radiologic data were extracted from the participants' medical records. The day of first positive swab for SARS-CoV-2 served as day 0 of illness for the study.

Glomerular filtration rate (GFR) was estimated using the Modified Diet in Renal Disease (MDRD) Equation. The MDRD equation enables GFR estimation from serum creatinine, and is more accurate for GFR $<60 \mathrm{ml} / \mathrm{min} / 1.73$ $\mathrm{m}^{2}$. Therefore, the cohort was divided into a CKD group (with eGFR $<60 \mathrm{ml} / \mathrm{min}$ ) and non-CKD (control) group (eGFR $>60 \mathrm{ml} / \mathrm{min}$ ). Estimated GFR (eGFR) was calculated for each patient using the baseline serum creatinine measurement.

\section{CKD stage}

CKD stage was defined as previously described [28]. Stage 3 included patients with eGFR $30-59 \mathrm{ml} / \mathrm{min}$, stage 4 eGFR $15-29(\mathrm{ml} / \mathrm{min})$ and stage 5 as GFR $<15(\mathrm{ml} / \mathrm{min})$. Patients treated with chronic dialysis were excluded.

Patients with eGFR $>60 \mathrm{~mL} / \mathrm{min}$, including stage 1 and 2 , served as the control group.

Since body surface area (BSA) was not available for this retrospective study, GFR was not corrected for BSA and was calculated in $\mathrm{mL} / \mathrm{min}$ without correction to BSA (1.73 $\left.\mathrm{m}^{2}\right)$ [29].

AKI was defined as greater than $30 \%$ increase in serum creatinine from baseline during COVID-19 infection [30].

\section{Variables}

Baseline clinical variables included eGFR, sex, weight $(\mathrm{kg})$, smoking history and comorbidities (chronic obstructive 
pulmonary disease, hypertension, diabetes mellitus, dyslipidemia, atrial fibrillation, ischemic heart disease, heart failure, peripheral vascular disease, previous stroke, malignancy, obesity).

Variables related to COVID-19 infection included clinical findings at presentation (COVID-19 symptoms and vital signs), laboratory findings (blood count, chemistry results, C-reactive protein and clotting tests), radiology findings from chest X-rays and computerized tomography studies, when performed and treatments given for COVID-19.

\section{Study endpoints}

The primary endpoint was in-hospital mortality after COVID-19 infection. Secondary outcomes included disease severity, respiratory deterioration and need for respiratory support (including oxygen), acute kidney injury, and need for renal replacement therapy.

Mortality data were collected from the National Social Security records.

COVID-19 disease severity was ranked according to National Institute of Health guidelines as asymptomatic, mild, moderate (with clinical or radiographic evidence of lower respiratory tract disease and oxygen saturation $\geq 94 \%$ while breathing room air), severe (saturation $<94 \%$, respiratory rate $>30 / \mathrm{min}$, infiltrates over $50 \%$ of lungs volume), or critical (requiring invasive or non-invasive ventilation, in shock, or organ failure) [1].

\section{Statistical analysis}

Descriptive statistics are presented as means with standard deviations and range, median or percentages. Variables between two study groups were compared using $t$ test, Mann-Whitney test, Fisher's exact test or chi-square, according to the scale of measured variables. $p$ values $<0.05$ were considered statistically significant. A multivariate logistic regression model including all significant variables in the univariate analysis was applied to estimate odds ratios (OR) for mortality. Survival curves were obtained using the Kaplan-Meier method and compared using two-sided log rank statistics. Data were analyzed using SPSS, Version 27 (IBM Corporation, Armonk, NY, USA).

\section{Ethical issues}

The study was approved by the Institutional Review Board of Meir Medical Center (no. MMC-351-20). The committee waived the requirement for informed consent due to the retrospective study design. The study was performed in accordance with the Declaration of Helsinki and Good Clinical Practice guidelines.

\section{Results}

A total of 658 patients were included, with a median age of 68 years (range 18-100). Mean follow-up was $108 \pm 84$ days after hospital admission due to COVID-19 infection.

Among 658 patients, 152 with eGFR $<60 \mathrm{ml} / \mathrm{min}$ were classified as the CKD group. The 506 patients with eGFR $>60 \mathrm{ml} / \mathrm{min}$ served as the control group. Figure 1 shows the distribution of the study cohort by CKD stage. Patients in the CKD group were older, with higher prevalence of hypertension, diabetes mellitus and atherosclerosis, as detailed in Table 1.

\section{COVID-19 infection}

The clinical presentation of the CKD and control groups were similar, except the CKD group had higher systolic blood pressure at hospital admission (Table 2). Baseline laboratory tests at admission revealed lower hemoglobin and higher C-reactive protein and D-dimer levels in the CKD group.

At presentation, there was no significant difference in disease severity between CKD stages ( $p=0.96$; Fig. 2$)$ and between the CKD and control groups $(p=0.984)$. Most CKD and control patients were determined to have mildto-moderate disease at admission, while $30.9 \%$ and $3.8 \%$ were defined as having severe and critical disease, respectively, with comparable distribution between CKD stages.

Baseline serum creatinine significantly correlated with serum creatinine at hospitalization $(r=0.638$,

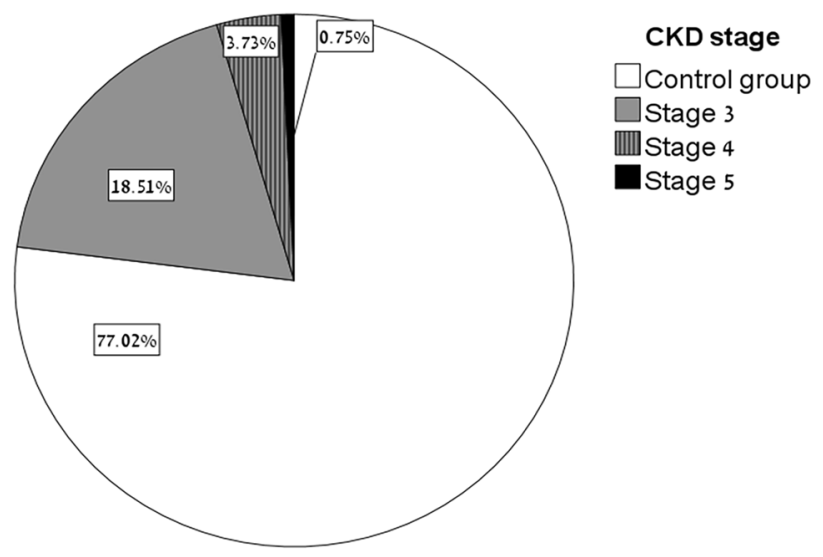

Fig. 1 Distribution of study cohort by CKD stage. 658 patients were included in this analysis, $77 \%$ had eGFR $>60 \mathrm{ml} / \mathrm{min}$ (Control group), $18.5 \%$ had eGFR 30-60 ml/min (CKD stage 3), $3.7 \%$ 15-30 $\mathrm{ml} / \mathrm{min}(\mathrm{CKD}$ stage 4$)$ and $<1 \%$ had GFR $<15 \mathrm{ml} / \mathrm{min}$ (stage $5)$ 
Table 1 Baseline characteristics of the study population
Table 2 Clinical presentation at admission to hospitalization

\begin{tabular}{lllr}
\hline Characteristic & CKD group $(n=152)$ & Control group $(n=506)$ & $p$ value \\
\hline Age (years) & $77.2 \pm 11.5$ & $62.3 \pm 16$ & $<0.001$ \\
eGFR (ml/min) & $44.9 \pm 12.6$ & $93.7 \pm 23.6$ & $<0.001$ \\
Male & $86(56.6 \%)$ & $288(56.9 \%)$ & 0.941 \\
Weight $(\mathrm{kg})$ & $77.9 \pm 18$ & $81.5 \pm 19.3$ & 0.095 \\
Body mass index & $28.2 \pm 5.6$ & $28.3 \pm 6.3$ & 0.818 \\
Obesity & $16(10.5 \%)$ & $58(11.5 \%)$ & 0.749 \\
Smoking & $15(9.9 \%)$ & $29(5.7 \%)$ & 0.073 \\
COPD & $10(6.6 \%)$ & $38(7.5 \%)$ & 0.699 \\
Hypertension & $107(70.4 \%)$ & $179(35.4 \%)$ & $<0.001$ \\
Diabetes & $69(45.4 \%)$ & $143(28.3 \%)$ & $<0.001$ \\
Dyslipidemia & $128(25.3 \%)$ & 0.005 \\
Malignancy & $56(36.8 \%)$ & $11(2.2 \%)$ & 0.74 \\
Atrial fibrillation & $4(2.6 \%)$ & $25(4.9 \%)$ & $<0.001$ \\
Coronary heart disease & $24(15.8 \%)$ & $53(10.5 \%)$ & $<0.001$ \\
Heart failure & $34(22.4 \%)$ & $13(2.6 \%)$ & $<0.001$ \\
PVD & $20(13.2 \%)$ & $57(11.3 \%)$ & $<0.001$ \\
Previous stroke & $40(26.3 \%)$ & $27(5.3 \%)$ & $<0.001$ \\
\hline
\end{tabular}

Values are presented as absolute numbers (percentage) or as mean $\pm \mathrm{SD}$

eGFR was calculated using MDRD formula as mentioned in the Methods section, using baseline serum creatinine before admission to hospitalization. Obesity was defined as BMI $>30$

$C K D$ chronic kidney disease, $C O P D$ chronic obstructive pulmonary disease, $P V D$ peripheral vascular disease

\begin{tabular}{lccc}
\hline Variable & CKD group $(n=152)$ & Control group $(n=506)$ & $p$-value \\
\hline Clinical presentation at hospital admission & & & \\
Temperature $\left({ }^{\circ} \mathrm{C}\right)$ & $37.1 \pm 0.66$ & $37.1 \pm 0.67$ & 0.422 \\
Heart rate (per minute) & $85.2 \pm 16.9$ & $86.1 \pm 15.1$ & 0.535 \\
Systolic blood pressure $(\mathrm{mmHg})$ & $137.4 \pm 26.1$ & $130.5 \pm 21.4$ & 0.002 \\
Diastolic blood pressure $(\mathrm{mmHg})$ & $71 \pm 14.9$ & $71.2 \pm 12.7$ & 0.891 \\
Oxygen saturation at room air & $91.8 \pm 9.2$ & $93.4 \pm 8.4$ & 0.198 \\
Laboratory values at hospital admission & & & \\
Serum creatinine $(\mathrm{mg} / \mathrm{dl})$ & $1.7 \pm 0.8$ & $1 \pm 0.5$ & $<0.001$ \\
C-reactive protein $(\mathrm{mg} / \mathrm{dl})$ & $12.9 \pm 9.9$ & $10.5 \pm 9.6$ & 0.014 \\
Hemoglobin $(\mathrm{g} / \mathrm{dl})$ & $12 \pm 1.9$ & $12.7 \pm 2$ & 0.001 \\
WBC $\left(\times 10^{9} / \mathrm{l}\right)$ & $7.5 \pm 3.2$ & $7.1 \pm 3.9$ & 0.266 \\
Abs. lymphocyte count $\left(\times 10^{9} / \mathrm{l}\right)$ & $1.1 \pm 0.6$ & $1.1 \pm 0.6$ & 0.915 \\
Abs. neutrophil count $\left(\times 10^{9} / \mathrm{l}\right)$ & $5.9 \pm 3$ & $5.5 \pm 3.8$ & 0.244 \\
NLR $($ per $\mu \mathrm{l})$ & $8.1 \pm 8.9$ & $7.3 \pm 8.5$ & 0.331 \\
Platelets $($ per $\mu \mathrm{l})$ & $219 \pm 99$ & $16.3 \pm 31.2$ & 0.73 \\
ALT (units/l) & $14.5 \pm 20.4$ & $37 \pm 86$ & 0.504 \\
AST (units/l) & $37.5 \pm 31.9$ & $1146 \pm 4025$ & 0.765 \\
Ferritin $(\mathrm{ng} / \mathrm{ml})$ & $811 \pm 847$ & $2687 \pm 6410$ & 0.383 \\
D-dimer $(\mathrm{ng} / \mathrm{ml})$ & $4118 \pm 9880$ & & 0.058 \\
\hline
\end{tabular}

$p<0.001)$, maximum serum creatinine during hospitalization $(r=0.64, p<0.001)$, CRP $(r=0.121, p=0.005)$, ferritin $(r=0.182, p<0.001)$ and troponin measurements $(r=0.373, p<0.001)$.
During hospitalization, more patients in the CKD group required oxygen support compared to the control group ( $55.9 \%$ vs. $31.8 \%$, respectively), OR for oxygen was 2.7 with 95\% CI 1.9-3.9, $p<0.001)$. However, they did not require 


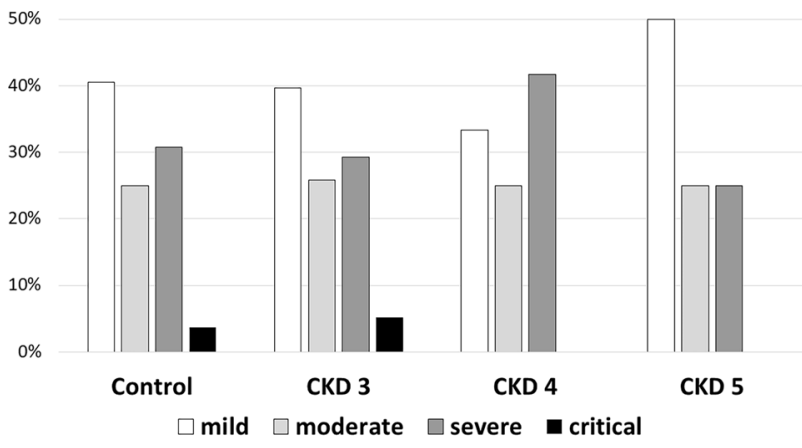

Fig. 2 Disease severity at different CKD stages at admission to hospital $(\mathrm{p}=0.96)$

more mechanical ventilation $(p=0.229)$. The CKD group did not require more hemodynamic support with vasopressors as compared to the control group (2.6\% vs. $1.4 \%$, $p=0.293$ ).

\section{AKI and renal outcomes}

Patients in the CKD group had baseline serum creatinine level of $1.7 \pm 0.8$ and eGFR of $44.9 \pm 12.6 \mathrm{ml} / \mathrm{min}$. Their CKD stage is shown in Fig. 1. Among the CKD group, $46.4 \%$ (70/151) patients had significant deterioration in kidney function during hospitalization with COVID-19 (AKI) vs. $63 / 483(13 \%)$ in the control group $(p<0.001)$.

The OR for acute kidney injury was 5.8 (95\%CI 3.8-8.7) in the CKD vs. control groups. The maximum serum creatinine level during hospitalization was $2 \pm 1.23 \mathrm{mg} / \mathrm{dl}$ in the CKD group.
Six patients $(3.9 \%)$ in the CKD group required renal replacement therapy vs. $6(1.2 \%)$ in the control group (OR $3.4(95 \%$ CI $1.1-10.8), p=0.026)$.

\section{Mortality}

In the CKD group, 32.2\% died after COVID-19 infection versus $14.8 \%$ in the control group (OR for mortality $=2.7$ (95\%CI 1.8-4.2), $p<0.001)$. Mortality increased as the CKD stage increased (14.8\% in non-CKD, $29.6 \%$ in CKD stage 3 , and $39.3 \%$ in CKD stages 4 and $5, p<0.001)$. A Kaplan-Meir curve shows that survival of CKD patients was significantly shorter compared to controls (Fig. 3, $p<0.001)$.

Among patients who died vs. survived after COVID-19 infection, higher CRP $(16.5 \pm 10.4$ vs. $9.8 \pm 9.1, p<0.001)$, ferritin $(2699.1 \pm 7596.2$ vs. $661.3 \pm 761.2, p<0.001)$, D-dimer $(10,470 \pm 15,099$ vs. $2521 \pm 4504, p<0.001)$ and troponin $(202.1 \pm 730.2$ vs. $24.6 \pm 80.6, p<0.001)$ respectively, were found.

Table 3 Multivariate logistic regression analysis model for predictors of mortality

\begin{tabular}{lllll}
\hline Variable & $\begin{array}{l}\text { Odds } \\
\text { ratio }\end{array}$ & \multicolumn{2}{l}{$95 \% \mathrm{CI}$} & $p$-value \\
\cline { 3 - 4 } & & Lower & Upper & \\
\hline CKD stage & 1.605 & 1.055 & 2.44 & 0.027 \\
Male sex & 2.082 & 1.42 & 3.054 & 0 \\
Diabetes mellitus & 0.909 & 0.612 & 1.35 & 0.637 \\
Hypertension & 1.345 & 0.909 & 1.989 & 0.138 \\
Age $>$ 75 years & 2.248 & 1.524 & 3.316 & 0 \\
Ischemic heart disease & 1.209 & 0.745 & 1.962 & 0.443 \\
\hline
\end{tabular}

Fig. 3 Survival of CKD patients was significantly worse as compared to control patients $(\mathrm{p}<0.001)$. Despite comparable follow-up from hospital admission, survival rate was significantly worse in the CKD group vs. control group, $\mathrm{p}<0.001)$

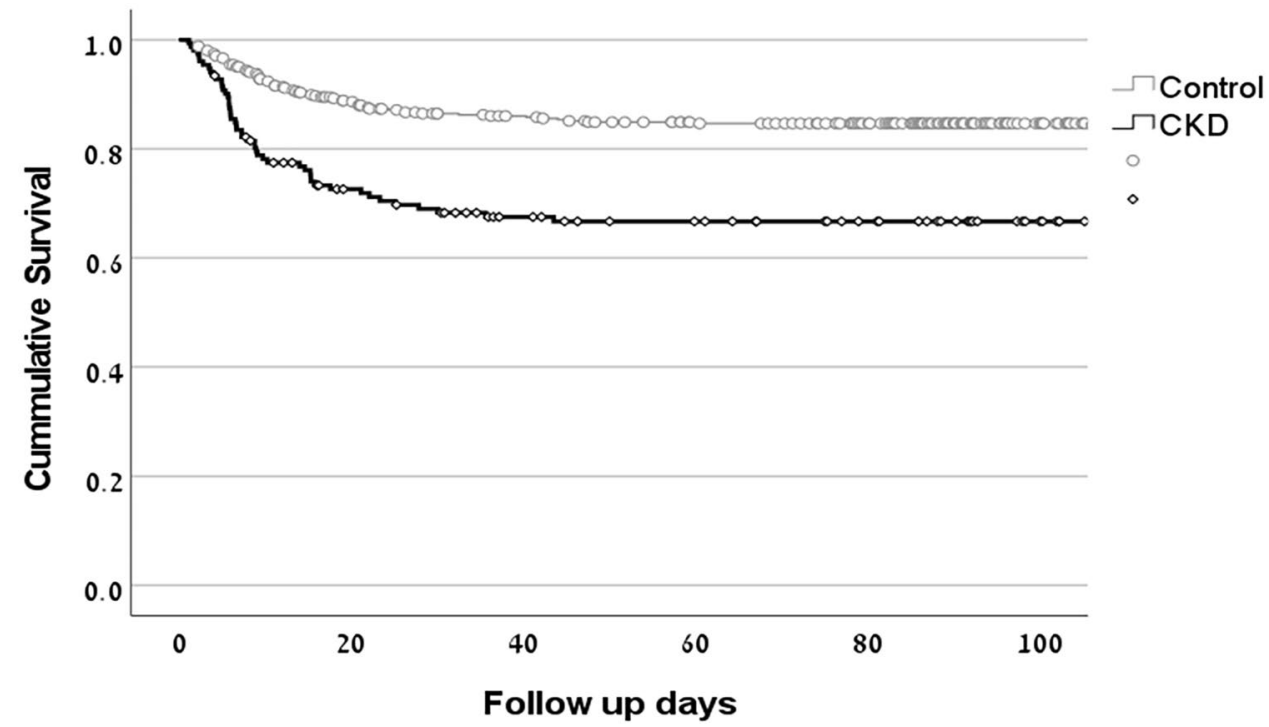


CKD, age and male sex were the most important predictors of mortality in a multivariate logistic regression analysis model (Table 3).

Among the patients who had AKI during hospitalization, $43.6 \%$ died compared to $12.6 \%$ of the patients without AKI $(p<0.001)$. OR for death among patients with AKI was 5.4 (95\% CI 3.5-8.3) in this cohort.

\section{Discussion}

This study assessed the impact of CKD on the clinical outcomes of unvaccinated patients infected with COVID-19. It included 658 patients with several measurements of kidney function, which enabled us to assess both baseline kidney function (eGFR and CKD stage) and AKI during hospitalization due to COVID-19 infection.

Despite comparable disease severity at presentation, patients with CKD had significantly greater instance of AKI and required more oxygen support and renal replacement therapy than control patients did. Mortality increased with higher CKD stage.

Patients in the CKD group were older, with higher prevalence of hypertension, diabetes mellitus and atherosclerosis. This is a predictable finding, as CKD is commonly comorbid with hypertension, diabetes, and cardiovascular disease and associated with several comorbidities [31].

Only a few studies that described CKD as a risk-factor for COVID-19 mortality, included information on CKD as a separate group of investigation. One study that compared three subgroups with CKD, found a graded association between the level of kidney dysfunction and the risk of COVID-19 mortality [24]. These mortality rates were higher than those of other high-risk groups, including obesity, hypertension and heart disease. Flythe et al. reported recently that dialysis and non-dialysis dependent CKD patients admitted to intensive care units had a higher risk for 28-day in-hospital death when compared to non-CKD patients [32]. These findings support our results that CKD is a condition associated with high mortality rate.

Laboratory data at admission revealed lower hemoglobin and higher C-reactive protein and D-dimer levels. These markers are known as indicators of severe infections and severe COVID-19 infection [13].

The CKD group had more AKI events, required more renal replacement therapy and experienced significant deterioration in kidney function during hospitalization for COVID-19. Risk factors associated with development of AKI among patients with acute respiratory distress syndrome were older age, severity of illness, diabetes and acidosis [33]. These are common comorbidities among the CKD population. AKI is a common complication with poor outcomes after COVID-19 infection. This can be aggravated among CKD patients, as CKD itself is recognized as a clear risk-factor for AKI [34-36]. In our study, disease severity at presentation was similar among all patients but during hospitalization the CKD group required more oxygen support, which means that their disease progressed and became more complicated.

Almost half (46.4\%) of the patients with CKD in our cohort experienced significant deterioration in kidney function (AKI) during hospitalization with COVID-19. This incidence is relatively high for hospitalized patients [37]. OR for death was 5.38 among patients who had AKI. These findings of high rate of AKI and its prognostic impact are supported by previous studies [14, 15]. Batlle et al. described an incidence of AKI ranging from 23 to $60 \%$. This was even higher among high-risk patients with COVID-19 infection, determined by findings of severe acute tubular necrosis, leucocyte infiltrates, endothelial damage, and ischemic and inflammatory changes in kidney histology [15].

The clinical implications of further studies in this field are very important. The pivotal finding of poor outcomes of COVID-19 infection among CKD patients is in accordance with previous reports $[35,36]$. Higher mortality rates, as well the need for more oxygen support and renal replacement therapy among the CKD group, indicate the need for special attention regarding COVID-19 infection. The presence of CKD should encourage physicians to initiate prompt therapeutic interventions, such as monoclonal antibodies and other special COVID-19 treatment options, especially in light of the increasing number of elderly CKD patients [38] and the aging of the population. Finally, emerging COVID19 variants that cost human life, even with the availability of vaccines, emphasize the importance of this study. Data about the relation between COVID-19 and CKD, and of CKD as a risk-factor are lacking. Additional research is needed to better understand the course of COVID-19 and risk factors for mortality among patients with CKD, especially after vaccination.

\section{Limitations}

There were several limitations to this study. First, as in any retrospective, record-based study, data collection was limited by inconsistent documentation and incomplete access to follow-up data, as some patients received follow-up in other healthcare facilities. Second, the data were collected before COVID-19 vaccinations were available and cannot be applied to vaccinated patients. Third, since the data were collected, the COVID-19 virus has developed new variants that affect its virulence and transmission, which influences disease severity and mortality. Finally, the diversity and heterogeneity of CKD patients, including their comorbidities, reduce the uniformity of the study population. 


\section{Conclusion}

CKD is common in the general population and its presence should encourage physicians to pay special attention to these patients who are infected with COVID-19, closely follow-up on their kidney function due to the increased risk for deterioration and oxygen desaturation, as well as other parameters of COVID-19 disease. Despite comparable disease severity at presentation, patients with CKD had significantly more AKI events and required more renal replacement therapy during hospitalization than control patients did. Mortality was increased among patients with higher CKD stages. We think that our findings are meaningful and should prompt vaccination of the CKD population against COVID-19.

Acknowledgements We thank Faye Schreiber, MS (Meir Medical Center) for editing the manuscript.

Funding This study was not funded. The authors declare that they have no relevant financial interests.

\section{Declarations}

Conflict of interest The authors declare that they have no conflicts of interest.

Ethical approval The study was approved by the Ethics Committee and Institutional Review Board of Meir Medical Center (no. MMC-35120). The committee waived the requirement for informed consent due to the retrospective design of the study. The study was performed in accordance with the Declaration of Helsinki and Good Clinical Practice guidelines.

\section{References}

1. National Center for Immunization and Respiratory Diseases (NCIRD) Division of Viral Diseases. Interim clinical guidance for management of patients with confirmed coronavirus disease (COVID-19). Cent Dis Control Prev. 2020;2019:1-10.

2. Pan L, Mu M, Yang P, Sun Y, Wang R, Yan J, et al. Clinical characteristics of COVID-19 patients with digestive symptoms in Hubei, China: a descriptive, cross-sectional, multicenter study. Am J Gastroenterol. 2020;115:766-73. https://doi.org/10.14309/ AJG.0000000000000620.

3. Giacomelli A, Pezzati L, Conti F, Bernacchia D, Siano M, Oreni $\mathrm{L}$, et al. Self-reported olfactory and taste disorders in patients with severe acute respiratory coronavirus 2 infection: a cross-sectional study. Clin Infect Dis. 2020;71:889-90. https://doi.org/10.1093/ CID/CIAA330.

4. Wu C, Chen X, Cai Y, Xia J, Zhou X, Xu S, et al. Risk factors associated with acute respiratory distress syndrome and death in patients with coronavirus disease 2019 pneumonia in Wuhan. China JAMA Intern Med. 2020;180:934-43. https://doi.org/10. 1001/JAMAINTERNMED.2020.0994.

5. Chen R, Liang W, Jiang M, Guan W, Zhan C, Wang T, et al. Risk factors of fatal outcome in hospitalized subjects with coronavirus disease 2019 from a nationwide analysis in China. Chest. 2020;158:97-105. https://doi.org/10.1016/j.chest.2020. 04.010.

6. Petrilli CM, Jones SA, Yang J, Rajagopalan H, O’Donnell L, Chernyak Y, et al. Factors associated with hospital admission and critical illness among 5279 people with coronavirus disease 2019 in New York City: prospective cohort study. BMJ. 2020. https:// doi.org/10.1136/bmj.m1966.

7. Williamson EJ, Walker AJ, Bhaskaran K, et al. Factors associated with COVID-19-related death using OpenSAFELY. Nature. 2020;584:430-6. https://doi.org/10.1038/s41586-020-2521-4.

8. Huang C, Wang Y, Li X, Ren L, Zhao J, Hu Y, et al. Clinical features of patients infected with 2019 novel coronavirus in Wuhan, China. Lancet. 2020;395:497-506. https://doi.org/10.1016/S01406736(20)30183-5.

9. Zhou F, Yu T, Du R, Fan G, Liu Y, Liu Z, et al. Clinical course and risk factors for mortality of adult inpatients with COVID19 in Wuhan, China: a retrospective cohort study. Lancet. 2020;395:1054-62. https://doi.org/10.1016/S0140-6736(20) 30566-3.

10. Wolff D, Nee S, Hickey NS, Marschollek M. Risk factors for Covid-19 severity and fatality: a structured literature review. Infection. 2021;49:15-28. https://doi.org/10.1007/ s15010-020-01509-1.

11. Zheng Z, Peng F, Xu B, Zhao J, Liu H, Peng J, et al. Risk factors of critical and mortal COVID-19 cases: a systematic literature review and meta-analysis. J Infect. 2020;81: e16. https://doi.org/ 10.1016/J.JINF.2020.04.021.

12. Booth A, Reed AB, Ponzo S, Yassaee A, Aral M, Plans D, et al. Population risk factors for severe disease and mortality in COVID19: a global systematic review and meta-analysis. PLoS ONE. 2021;16:1-30. https://doi.org/10.1371/journal.pone.0247461.

13. Zhou F, Yu T, Du R, Fan G, Liu Y, Liu Z, Xiang J, et al. Clinical course and risk factors for mortality of adult inpatients with COVID-19 in Wuhan, China: a retrospective cohort study. Lancet (London, England). 2020;395:1054-62. https://doi.org/10.1016/ S0140-6736(20)30566-3.

14. Ng JH, Bijol V, Sparks MA, Sise ME, Izzedine H, Jhaveri KD. Pathophysiology and pathology of acute kidney injury in patients with COVID-19. Adv Chronic Kidney Dis. 2020;27:365-76. https://doi.org/10.1053/J.ACKD.2020.09.003.

15. Batlle D, Soler MJ, Sparks MA, Hiremath S, South AM, Welling PA, et al. Acute kidney injury in COVID-19: emerging evidence of a distinct pathophysiology. J Am Soc Nephrol. 2020;31:13803. https://doi.org/10.1681/ASN.2020040419.

16. Pan X, Xu D, Zhang H, Zhou W, Wang L, Cui X. Identification of a potential mechanism of acute kidney injury during the COVID19 outbreak: a study based on single-cell transcriptome analysis. Intensive Care Med. 2020;46:1114-6. https://doi.org/10.1007/ S00134-020-06026-1.

17. Buonaguro FM, Ascierto PA, Morse GD, Buonaguro L, Puzanov I, Tornesello ML, et al. Covid-19: time for a paradigm change. Rev Med Virol. 2020;30: e2134. https://doi.org/10.1002/RMV. 2134.

18. Henderson LA, Canna SW, Schulert GS, Volpi S, Lee PY, Kernan KF, et al. On the ALERT FOR CYTOKINE STORM: IMMUNOPATHOLOGy in COVID-19. Arthritis Rheumatol. 2020;72:1059-63. https://doi.org/10.1002/ART.41285.

19. Li H, Liu L, Zhang D, Xu J, Dai H, Tang N, et al. SARSCoV-2 and viral sepsis: observations and hypotheses. Lancet. 2020;395:1517-20. https://doi.org/10.1016/S0140-6736(20) 30920-X.

20. Tang N, Bai H, Chen X, Gong J, Li D, Sun Z. Anticoagulant treatment is associated with decreased mortality in severe coronavirus disease 2019 patients with coagulopathy. J Thromb Haemost. 2020;18:1094-9. https://doi.org/10.1111/JTH.14817. 
21. Terpos E, Ntanasis-Stathopoulos I, Elalamy I, Kastritis E, Sergentanis TN, Politou M, et al. Hematological findings and complications of COVID-19. Am J Hematol. 2020;95:834-47. https://doi. org/10.1002/AJH.25829.

22. Cheng Y, Luo R, Wang K, Zhang M, Wang Z, Dong L, et al. Kidney impairment is associated with in-hospital death of COVID19 patients. MedRxiv. 2020. https://doi.org/10.1101/2020.02.18. 20023242.

23. Henry BM. Lippi G (2020) Chronic kidney disease is associated with severe coronavirus disease 2019 (COVID-19) infection. Int Urol Nephrol. 2020;52:1193-4. https://doi.org/10.1007/ S11255-020-02451-9.

24. Gansevoort RT, Hilbrands LB. CKD is a key risk factor for COVID-19 mortality. Nat Rev Nephrol. 2020;1998:19-20. https:// doi.org/10.1038/s41581-020-00349-4.

25. Fernández-Fresnedo G, Ramos MA, González-Pardo MC, De Francisco ALM, López-Hoyos M, Arias M. B lymphopenia in uraemia is related to an accelerated in vitro apoptosis and dysregulation of Bcl-2. Nephrol Dial Transplant. 2000;15:502-10. https://doi.org/10.1093/ndt/15.4.502.

26. Ahmadian E, Khatibi SMH, Soofiyani SR, Abediazar S, Shoja MM, Ardalan M, et al. Covid-19 and kidney injury: Pathophysiology and molecular mechanisms. Rev Med Virol. 2021;31: e2176. https://doi.org/10.1002/RMV.2176.

27. Thomas R, Kanso A, Sedor JR. Chronic kidney disease and its complications. Prim Care. 2008;35:329. https://doi.org/10.1016/J. POP.2008.01.008.

28. Levin A, Stevens PE. Summary of recommendation statements. Kidney Int Suppl. 2013;3:5. https://doi.org/10.1038/KISUP.2012. 77.

29. Schwandt A, Denkinger M, Fasching P, Pfeifer M, Wagner C, Weiland J, Zeyfang A, Holl RW. Comparison of MDRD, CKD-EPI, and Cockcroft-Gault equation in relation to measured glomerular filtration rate among a large cohort with diabetes. J Diabetes Complicat. 2017;31:1376-83. https://doi.org/10.1016/J.JDIACOMP. 2017.06.016
30. Makris K, Spanou L. Acute kidney injury: definition, pathophysiology and clinical phenotypes. Clin Biochem Rev. 2016;37:85.

31. MacRae C, Mercer SW, Guthrie B, Henderson D. Comorbidity in chronic kidney disease: a large cross-sectional study of prevalence in Scottish primary care. Br J Gen Pract. 2021;71:e243-9. https:// doi.org/10.3399/BJGP20X714125.

32. Flythe JE, Assimon MM, Tugman MJ, Chang EH, Gupta S, Shah $\mathrm{J}$, et al. Characteristics and outcomes of individuals with preexisting kidney disease and COVID-19 admitted to intensive care units in the United States. Am J Kidney Dis. 2021;77:190-203.e1. https://doi.org/10.1053/j.ajkd.2020.09.003.

33. Panitchote A, Mehkri O, Hastings A, Hanane T, Demirjian S, Torbic $\mathrm{H}$, et al. Factors associated with acute kidney injury in acute respiratory distress syndrome. Ann Intensive Care. 2019;9:1-10. https://doi.org/10.1186/S13613-019-0552-5.

34. Hsu RK, Hsu C. The role of acute kidney injury in chronic kidney disease. Semin Nephrol. 2016;36:283. https://doi.org/10.1016/J. SEMNEPHROL.2016.05.005.

35. Council E-E, Ortiz A, Cozzolino M, Fliser D, Fouque D, Goumenos D, et al. Chronic kidney disease is a key risk factor for severe COVID-19: a call to action by the ERA-EDTA. Nephrol Dial Transplant. 2021;36:87-94. https://doi.org/10.1093/NDT/ GFAA314.

36. Gok M, Cetinkaya H, Kandemir T, et al. Chronic kidney disease predicts poor outcomes of COVID-19 patients. Int Urol Nephrol. 2021;53(9):1891-8. https://doi.org/10.1007/s11255-020-02758-7.

37. Kellum JA, Romagnani P, Ashuntantang G, et al. Acute kidney injury. Nat Rev Dis Primers. 2021;7:52. https://doi.org/10.1038/ s41572-021-00284-Z.

38. Lv JC, Zhang LX. Prevalence and disease burden of chronic kidney disease. Adv Exp Med Biol. 2019;1165:3-15. https://doi.org/ 10.1007/978-981-13-8871-2_1.

Publisher's Note Springer Nature remains neutral with regard to jurisdictional claims in published maps and institutional affiliations. 